\title{
Transluminal endovascular aortic repair and pregnancy: a case report
}

\author{
Duiella SF ${ }^{*}$, Gaia G', Bonanomi C ${ }^{2}$, Ossola MW'1, Rossi G ${ }^{1}$ and Fedele $\mathrm{L}^{1}$ \\ ${ }^{1}$ Department of Obstetrics and Gynecology, Fondazione IRCCS Ca' Granda Ospedale Maggiore Policlinico, Italy \\ ${ }^{2}$ Department of Cardiology, Fondazione IRCCS Ca' Granda Ospedale Maggiore Policlinico, Italy
}

\begin{abstract}
Acute Traumatic Aortic Transection (ATAT) is a dramatic event related to extremely high mortality. Transluminal Endovascular Aortic Repair (TEVAR) is currently the preferred Strategy for such lesions and offers an alternative to open surgical repair.

In this article we discuss the case of a 21-year old woman who suffered from an ATAT of the aortic isthmus along with multiple fractures and visceral lesions during a car accident. Three years after the endovascular repair of the aorta our patient became pregnant. The pregnancy evolved without any complications, but since no spontaneous labour occurred, and the patient refused labour induction, we decided to perform an elective caesarean section at 41 week. To our knowledge, this is the first case described in literature of a patient undergoing a caesarean section after TEVAR for ATAT.
\end{abstract}

\section{Introduction}

Acute traumatic transection of the aorta is a high mortality related event, burdened by the multiple traumatism frequently associated (such as abdominal and pelvic lesions or head trauma). The aortic rupture is due to the displacement of the aortic arch as a result of a rapid deceleration.

Isthmic lesions occur more commonly (50-70\%), followed by ascending aorta and aortic arch lesions (18\%) and finally descending aorta lesions (14\%).

In order to decrease the extremely high mortality rate associated to this traumatic event is necessary an immediate diagnosis and endovascular treatment (within 24 hours). However up to $85-90 \%$ of these patients are reported to die at the time of the injury and around 20-30\% die during surgery or diagnostic procedure.

Currently the diagnostic gold standard procedure is considered to be the angio-TC.

Regarding the treatment approach Transluminal Endovascular Aortic Repair (TEVAR) has become the preferred strategy for such lesions and offers an alternative to open surgical repair, which seems to be associated to a higher mortality rate and procedure related complications.

Due to the high mortality and frequent complications related to this event, pregnancy is rare in these patients; currently, according to the scientific literature, only one case of uncomplicated vaginal delivery was described. Herein we describe the second case of a patient with an uncomplicated pregnancy ended with a caesarean section.

\section{Case report}

The patient, after a multiple trauma occurred in a car accident at the age of 21, suffered of an ATAT of the aortic isthmus with massive left hemothorax, multiple hepatic, splenic and renal lesions, fracture of the V finger of the left hand, cleft of multiple sacral vertebras; the sacrum was fractured and the sacroiliac joint was injured on both sides.

After admission, the patient was immediately treated with endovascular aortic repair; a Bolton Relay 22x100 endoprothesis was positioned without any complications. She was discharged after 12 days with antiplatelet therapy and has fully recovered from the multiple injuries.

Thoraco-abdominal angio computered tomography was performed annually and no endoleak neither any other site of aortic dissection was documented.

Three years later, the patient became pregnant and was followed by a multidisciplinary team throughout the pregnancy.

Antiplatelet therapy with Cardioaspirin 100mg was continued. At 12, 24 and 36 weeks obstetric clinical evaluation and fetal monitoring was performed and the cardiologist controlled the patient's clinical parameter (blood pressure BP, heart rate HR), EKG and Transthoracic Echocardiography (TTE).

During the pregnancy, she was followed up in our department by a team of obstetricians, cardiologist, vascular surgeon and anesthesiologist.

CT scan was performed one month before the patient became

Correspondence to: Duiella Silvia Francesca, Department of Obstetrics and Gynecology Fondazione IRCCS Ca'Granda Ospedale Maggiore Policlinico, Via Commenda 12, 20100 Milano MI, Italy, Tel: +39 02 55032218; Fax: +39 02 55032289; E-mail: silviafrancescaduiella@gmail.com

Key words: aortic stent graft, acute traumatic aortic transaction, pregnancy, caesarean section

Received: February 15, 2015; Accepted: March 08, 2015; Published: March 11, 2015 
pregnant and documented good results of the endovascular procedure; so no further exams were carried out.

The pregnancy evolved without any significant complication; fetal ultrasound scan showed a normal anatomy and growth; EKG and TTE were regular.

Blood pressure values were always into normal range and, in spite of the physiological increase of stroke volume; no pathological gradients were tested by CW Doppler through the aortic STENT- graft at the isthmus.

In order to optimize timing and mode of delivery the patient was admitted to our Department at 36 weeks of gestation.

The patient clearly preferred a vaginal delivery over a caesarean section without any kind of induction; so timing and modality of delivery was discussed by the team of obstetricians, anesthesiologists, cardiologists and vascular surgeons and no major contraindications were posed over a vaginal delivery with adequate peridural anesthesia.

The patient was discharged with the following therapeutic program:

- obstetrical follow up according to current diagnostic protocols

- hospitalization planned at 41 weeks of gestation to perform a caesarean section in case of failure to onset of spontaneous labour.

- Cardioaspirine 100mg was replaced by low molecular weight Heparine 0,4 at 37 weeks of gestation.

Due to the failure of an onset of spontaneous labour a caesarean section was performed at $41+2$ gestational weeks; spinal anesthesia wad used and antibiotic prophylaxis for endocarditis, with Amoxicillin $2 \mathrm{~g}$ and Gentamicin $80 \mathrm{mg}$, was administrated during surgery.

In order to reduce the impact of the possible hemodynamic changes and the increase of the aortic pressure, caused by pressure applied to the uterine fundus, a profilactic vacuum extraction was performed using Kiwi Omnicup during the delivery.

Delivery took place without any complications, overall blood loss was estimated about $900 \mathrm{ml}$; operatory time was 35 minutes. The baby was an healthy female and weighed $3900 \mathrm{~g}$.

Post-partum was uneventful and breast feeding was successfully started.
Moreover antibiotic therapy with Cefuroxime 500mg orally, 1gr/ day for 7 days and antitromboembolic prophylaxis with LMWH $0.4 \mathrm{ml} \mathrm{sc}, 1 \mathrm{fl} /$ day for 16 days were carried out. Antiplatelet therapy was restarted the day after surgery.

The cardiological examination showed good hemodynamic compensation and absence of cardiac complications.

The patient was discharged from the hospital four days after surgery. The puerperium has elapsed regularly.

\section{Discussion}

According to the literature this is the first case described of a patient with TEVAR for ATAT who underwent to caesarean section at 41 weeks of gestational age, after a physiological pregnancy [1]

Pregnancy in patients survivors after ATAT is very rare because of the extremely high mortality rate associated to this traumatic event (up to $85-90 \%$ of these patients die at the time of the injury and $20-30 \%$ during surgery or diagnostic procedure) [2].

Shelechta recently described a similar case of pregnancy after TEVAR and concluded that vaginal delivery may be possible if the stent graft is in regular position and the rest of the aorta is free from dissection or aneurysm formation [1].

This case contributes to remark that the aortic endoprothesis supports the increase of blood volume and aortic pressure that featured pregnancy, maintaining a framework of hemodynamic stability during the caesarean section.

Careful obstetrical and cardiovascular follow up is recommended during the pregnancy in order to identify in advance possible incoming complications.

More experience is necessary to confirm our findings in order to detect the optimal mode of delivery in such patients; moreover any experience was reported in complicated pregnancies.

\section{References}

1. Schlechta B, Wiedemann D, Eppel W, Kocher A (2012) Uncomplicated vaginal delivery 6 years after stent graft repair of an acute traumatic aortic transection. Interact Cardiovasc Thorac Surg 14: 120-121. [Crossref]

2. Plummer D, Petro K, Akbari C, O’Donnel S (2006) Endovascular repair of traumatic thoracic aortic disruption. Perspect Vasc Surg Endovasc Ther 18: 132. [Crossref]

Copyright: (C2015 Duiella SF. This is an open-access article distributed under the terms of the Creative Commons Attribution License, which permits unrestricted use, distribution, and reproduction in any medium, provided the original author and source are credited. 